\title{
Perianal Pediatric Crohn Disease Is Associated With a Distinct Phenotype and Greater Inflammatory Burden
}

\author{
${ }^{*}$ Amit Assa, ${ }^{\dagger}$ Michal Amitai, ${ }^{\ddagger \S}$ Mary-Louise Greer, ${ }^{\ddagger \S}$ Denise A Castro, ${ }^{\|}$Ruth C. Kuint, \\ ${ }^{\top}$ Maria Martínez-León, ${ }^{\#}$ Izabela Herman-Sucharska, ${ }^{* *}$ Eva Coppenrath, ${ }^{\dagger \dagger}$ Sudha Anupindi, \\ ${ }^{\ddagger}$ Alexander Towbin, ${ }^{\S \S}$ Douglas Moote, ${ }^{\prime|l|}$ Osnat Konen, "Li-tal Pratt, ${ }^{\# \#}$ Anne Griffiths, \\ and ${ }^{* * *}$ Dan Turner, on behalf of the ImageKids Study Group
}

\begin{abstract}
Objectives: Data on the outcomes of children with perianal Crohn disease $(\mathrm{pCD})$ are limited, although its presence is often used for justifying early use of biologics. We aimed to assess whether pCD in children is associated with more severe outcomes as found in adults.

Methods: Data were extracted from the ImageKids database, a prospective, multicenter, longitudinal cohort study. The study enrolled 246 children at disease onset or thereafter. All patients underwent comprehensive clinical, endoscopic, and radiologic evaluation at enrollment; 98 children had repeat evaluation at 18 months.

Results: Of the 234 included patients (mean age $14.2 \pm 2.4$ years; 131 [56\%] boys), 57 (24\%) had perianal findings, whereas only 21 (9\%) had fistulizing perianal disease. Children with $\mathrm{pCD}$ had reduced weight and height $z$ scores compared with non-pCD patients ( -0.9 vs $-0.35, P=0.03$ and -0.68 vs -0.23 , respectively; $P=0.04$ ), higher weighted pediatric $\mathrm{CD}$ activity index (32 [interquartile range $16-50$ ] vs 20 [8-37]; $P=0.004$ ), lower serum albumin (3.6 \pm 0.7 vs $4.5 \pm 0.8, P=0.016)$, and higher magnetic resonance enterography global inflammatory score $(P=0.04)$. Children with pCD had more rectal ( $57 \%$ vs $38 \%, P=0.04)$, and jejunal involvement $(31 \%$ vs $11 \%$ $P=0.003)$ and a higher prevalence of granulomas ( $64 \%$ vs $23 \%, P=0.0001)$. Magnetic resonance enterography-based damage scores did not differ between groups. Patients with skin tags/fissures only, had similar clinical, endoscopic, and radiologic characteristics as patients with no perianal findings. Conclusions: Pediatric patients with $\mathrm{pCD}$ with fistulizing disease have distinct phenotypic features and a predisposition to a greater inflammatory burden.
\end{abstract}

Key Words: children, fistula, growth, penetrating, stricturing

(JPGN 2017;65: 293-298)

Received October 17, 2016; accepted November 24, 2016.

From the *Institute of Gastroenterology, Nutrition and Liver Disease, Schneider Children's Hospital, Petah Tikva, Sackler School of Medicine, Tel Aviv University, Tel Aviv, Israel, the tDepartment of Radiology, Sheba Medical Center, Tel-Hashomer, Israel, the $\ddagger$ Department of Diagnostic Imaging, Hospital for Sick Children, the $\S$ Department of Medical Imaging, University of Toronto, Toronto, Ontario, Canada, the IPediatric Radiology Unit, Shaare Zedek Medical Center, Jerusalem, Israel, the Department of Radiology, Hospital Regional Universitario Carlos Haya, Malaga, Spain, the \#Electroradiology Department, Faculty of Health Sciences, Collegium Medicum, Jagiellonian University, Cracow, Poland, the $* *$ Department of Radiology, University of Munich, Munich, Germany, the ††Department of Radiology, The Children's Hospital of Philadelphia, Philadelphia, PA, the $\ddagger$ Department of Radiology, Cincinnati Children's Hospital, Cincinnati, OH, the $\S \S$ Department of Radiology, Connecticut Children's Medical Center, Hartford, CT, the |||lDepartment of Radiology, Schneider Children's Hospital, Petah

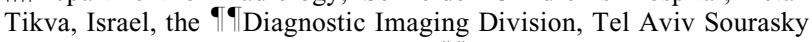
Medical Center, Tel Aviv, Israel, the \#\#Division of Gastroenterology, Hospital for Sick Children, Toronto, Ontario, Canada, and the

\section{What Is Known}

- Perianal Crohn disease occurs in approximately $30 \%$ of patients over the course of disease.

- Adult studies showed that perianal disease is associated with luminal complications.

- Data on the phenotypic features and natural history of perianal Crohn disease are scarce.

\section{What Is New}

- Patients with perianal Crohn disease have a greater inflammatory burden.

- Nevertheless, we did not observe higher rates of luminal damage associated with this phenotype.

- Isolated noninflamed skin tags and/or fissures are not associated with severe phenotype, and thus may not require a radiologic evaluation or treatment escalation.

A pproximately $25 \%$ of patients with irritable bowel disease are diagnosed during childhood or adolescence (1). Pediatric onset Crohn disease (CD) is generally associated with a more aggressive course, including a higher rate of complications and a need for

***Institute of Gastroenterology, Nutrition and Liver Disease, Shaare Zedek Medical Center, Jerusalem, Israel.

Address correspondence and reprint requests to Amit Assa, MD, MHA, Institute of Gastroenterology, Nutrition and Liver Disease, Schneider Children's Medical Center of Israel, Sackler Faculty of Medicine, Tel Aviv University, 14 Kaplan St., Petah Tikva 4920235, Israel (e mail: dr.amit.assa@gmail.com).

Supplemental digital content is available for this article. Direct URL citations appear in the printed text, and links to the digital files are provided in the HTML text of this article on the journal's Web site (www.jpgn.org).

The ImageKids study has been funded by an educational grant from Abbvie International.

Drs Griffiths and Turner contributed equally to the study.

The authors report no conflicts of interest.

Copyright (c) 2016 by European Society for Pediatric Gastroenterology, Hepatology, and Nutrition and North American Society for Pediatric Gastroenterology, Hepatology, and Nutrition

DOI: 10.1097/MPG.0000000000001484 
intensified medical therapy $(2,3)$. Perianal involvement, including inflamed skin tags, fistulae, and abscesses is a common, often debilitating manifestation of CD occurring in approximately $30 \%$ of patients over the course of disease $(4,5)$. Studies in adult patients with $\mathrm{CD}$ have shown that perianal disease is associated with a more rapid progression from inflammatory to stricturing or penetrating phenotype, which mandates intensified treatment strategy at disease onset, often with biologics (6-9). Similar data in children are scarce although younger adult patients were found to be at the greatest risk for $\mathrm{pCD}$ (6). A recent report indicated that children with $\mathrm{CD}$ who develop perianal fistulae are more likely to require diverting ileostomy or colectomy compared with those who do not present with perianal disease (10). Despite the limited pediatric data and mainly extrapolating from adult literature, European Crohn's and Colitis Organisation/European Society for Paediatric Gastroenterology Hepatology and Nutrition guidelines for managing pCD state that the presence of severe perianal disease is a predictive factor for poor outcome, justifying more aggressive treatment (11).

We therefore aimed to assess whether a perianal phenotype is associated with more severe outcomes in pediatric $\mathrm{CD}$, and to explore clinical, laboratory, endoscopic, and radiologic factors associated with this phenotype on the prospective ImageKids database.

\section{MATERIALS AND METHODS}

\section{Setting and Design}

Data were extracted from the ImageKids database, which is a longitudinal, prospective, multicenter cohort study aimed to develop and evaluate pediatric magnetic resonance enterography (MRE)-based indices-one of inflammation and one of intestinal damage. In this ancillary study, we stratified the patients according to those with and those without perianal disease at enrollment. Perianal disease was defined as any perianal finding including skin tags, fissures, fistulae, or abscess either by physical examination or by pelvic magnetic resonance imaging (pMRI). Fistulizing perianal disease was defined as a fistula and/or abscess diagnosed by pMRI.

\section{Patients}

The ImageKids study enrolled 246 children from 5 to 18 years of age with established diagnosis of $\mathrm{CD}$ by the presence of accepted clinical, radiologic, endoscopic, and histologic criteria (11). Children were included at any phase of the disease (at diagnosis and thereafter) according to predefined stratification: $20 \%$ within 3 months of diagnosis, $20 \%$ between 3 months and 2 years, $20 \%$ between 2.01 to 3 years and $40 \%$ more than 3 years from diagnosis. Patients unable to tolerate MRE were excluded. The first 120 enrolled children were followed for 18 months when MRE and clinical evaluation were repeated. The present study uses both the baseline visit and a subgroup of patients who completed the 18 months' follow-up visit.

At enrollment, all patients underwent ileocolonoscopy, esophagogastroduodenoscopy, and MRE as part of clinical care. pMRI was performed in all patients unless the perianal area was completely normal on physical examination and history taking (ie, no current or previous fissures, skin tags, fistulae, or perianal abscess).

\section{Description of Variables}

Explicit demographic, clinical, laboratory, therapeutic, anthropometric, endoscopic, and radiologic data were recorded at enrollment. Data were entered to a Web-based electronic case report file. Because of different reference values at different centers, C-reactive protein was analyzed as a categorical variable (normal/abnormal) rather than an absolute number. Disease activity was assessed using the weighted Pediatric CD Activity Index (wPCDAI) and Physician Global Assessment (PGA) using both categorical analysis (0-3; 0: quiescent, 1 : mild, 2 : moderate, 3 : severe) and a $100 \mathrm{~mm}$ visual analog scale (in which $0 \mathrm{~mm}$ means quiescent disease and $100 \mathrm{~mm}$ severe). Disease phenotype at diagnosis was categorized according to the Paris classification (12). Height, weight, and body mass index were converted to age- and sex-adjusted standard deviation scores ( $z$ scores), using the World Health Organization (Anthro and Anthro plus software for personal computers, version 2, 2007: Software for assessing growth and development of the world's children. Geneva: WHO, 2007 [http://www.who.int/childgrowth/software/en/]). Therapies and surgical interventions from the time of diagnosis to enrollment were retrieved from medical charts.

MRE and pMRI were assessed by 2 experienced radiologists who also provided global assessments on the MRE examinations for both inflammation and damage separately on a $100 \mathrm{~mm}$ visual analog scale (in which $0 \mathrm{~mm}$ means no inflammation/damage and $100 \mathrm{~mm}$ severe). Degree of mucosal inflammation and stenosis was recorded on ileocolonoscopy and esophagogastroduodenoscopy prospectively, using 2 scoring systems; a global assessment of none, mild, moderate, and severe, at each endoscopic segment (terminal ileum, ascending colon, transverse colon, descending colon, rectosigmoid, esophagus, stomach, and duodenum) and by using the simple endoscopic score for $\mathrm{CD}$, at each intestinal segment (both in the ileocolon and in the upper gastrointestinal [UGI] tract (13)).

\section{Data Analyses}

Data are reported as proportions (\%), means \pm standard deviations, or medians (interquartile range), as appropriate. Continuous data were compared using Student $t$ test, or the Wilcoxon rank sum test, as appropriate, for the distribution normality. Categorical variables were compared using chi-square or Fisher exact, as appropriate, and were reported as frequency and percentage. Multivariate logistic regression model was then sought for analyzing the association between perianal phenotype and selected variables by relevance, including those found by a univariate analysis with significance of $<0.1$. The first block in each regression included age, sex, and pCD. The second block included potential confounders, which were selected for inclusion in the regression using the forward method.

For the longitudinal analysis Wilcoxon and McNemar tests were used to compare parameters between enrollment and end of follow-up. Generalized estimating equations and linear mixed model were used to assess interactions for the 3 groups (no perianal findings, skin tags/fissures only, and perianal fistulizing disease) between the 2 time points while controlling for age, sex, and time for diagnosis. $P<0.05$ was considered as statistically significant unless stated otherwise. SPSS version 23 (IBM Corp, Armonk, NY) was used for all statistical analyses. The study protocol was approved by each participating site local institutional review board.

\section{RESULTS}

Of the 246 enrolled children, 12 had missing perianal data and thus 234 were included in the present study (Table 1). Fifty-six patients were enrolled at diagnosis, 50 patients were between 3 months to 2 years after diagnosis and 128 patients were enrolled with longer disease duration.

Fifty-seven patients (24\%) had perianal involvement on physical examination: 40 of $234(17 \%)$ with skin tags or fissures, whereas 8 of $40(20 \%)$ were also inflamed. All 57 patients underwent pMRI. Three of the 8 patients with inflamed skin tags $(37 \%)$ had fistulae documented in pMRI compared with only one of 32 of $40(3 \%)$ with noninflamed skin tags or fissures 


\begin{tabular}{|c|c|c|c|}
\hline & Nonperianal $(\mathrm{n}=177)$ & Perianal $(\mathrm{n}=57)$ & Significance $(P)$ \\
\hline Female, n (\%) & $78(44 \%)$ & $25(44 \%)$ & 0.9 \\
\hline Age at diagnosis (y), mean (SD) & $11.5 \pm 3.3$ & $11.9 \pm 2.5$ & 0.1 \\
\hline Age at enrollment (y), mean (SD) & $14.2 \pm 2.6$ & $14.1 \pm 2.1$ & 0.8 \\
\hline Disease duration $(\mathrm{y})$, mean $(\mathrm{SD})$ & $2.4(0.6-2.5)$ & $2(0.5-3.5)$ & 0.1 \\
\hline \multicolumn{4}{|l|}{ Anthropometric measurements, median (IQR) } \\
\hline Weight $z$ score & $-0.35(-1.3-0.5)$ & $-0.9(-1.9-0.2)$ & 0.03 \\
\hline Height $z$ score & $-0.23(-1.1-0.6)$ & $-0.68(-1.4-0.2)$ & 0.04 \\
\hline BMI $z$ score & $-0.35(-1.3-0.4)$ & $-0.77(-1.4-0.2)$ & 0.17 \\
\hline \multicolumn{4}{|l|}{ Disease location, n (\%) } \\
\hline Ileal (L1) & $70(40 \%)$ & $14(25 \%)$ & 0.08 \\
\hline Colonic (L2) & $33(18 \%)$ & $10(18 \%)$ & \\
\hline Ileocolonic (L3) & $74(42 \%)$ & $33(57 \%)$ & \\
\hline Upper GI (L4) & $70 / 157(45 \%)$ & $28 / 52(56 \%)$ & 0.1 \\
\hline \multicolumn{4}{|l|}{ Disease behavior, $\mathrm{n}(\%)$} \\
\hline Inflammatory (B1) & $122(69 \%)$ & $39(68 \%)$ & 0.6 \\
\hline Stricturing (B2) & $49(28 \%)$ & $14(25 \%)$ & \\
\hline Penetrating (B3) & $6(3 \%)$ & $4(7 \%)$ & \\
\hline Family history of IBD (first-degree relative), n (\%) & $32(18 \%)$ & $13(23 \%)$ & 0.2 \\
\hline Granuloma in histology, $\mathrm{n}(\%)$ & $39 / 167(23 \%)$ & $35 / 55(64 \%)$ & 0.0001 \\
\hline ESR, mean (SD) & $21 \pm 17$ & $30 \pm 19$ & 0.04 \\
\hline Platelets, mean (SD) & $353 \pm 122$ & $386 \pm 133$ & 0.06 \\
\hline Albumin, $(\mathrm{g} / \mathrm{dL})$, mean $(\mathrm{SD})$ & $4.5 \pm 0.8$ & $3.6 \pm 0.7$ & 0.016 \\
\hline Hemoglobin (g/dL), mean (SD) & $12.7 \pm 1.5$ & $11.8 \pm(1.3)$ & 0.15 \\
\hline Abnormal CRP, n (\%) & 77/165 (47\%) & $35 / 55(60 \%)$ & 0.15 \\
\hline PGA disease activity (moderate-severe),n (\%) & $64(36 \%)$ & $34(60 \%)$ & 0.02 \\
\hline Cecal inflammation, $\mathrm{n}(\%)$ & $55 / 116(47 \%)$ & $27 / 36(75 \%)$ & 0.02 \\
\hline Rectal inflammation, $\mathrm{n}(\%)$ & $67 / 176(38 \%)$ & $32 / 56(57 \%)$ & 0.04 \\
\hline Jejunal inflammation, n (\%) & $18 / 162(11 \%)$ & $17 / 54(31 \%)$ & 0.003 \\
\hline Surgical resection, $\mathrm{n}(\%)$ & $9(5 \%)$ & $4(7 \%)$ & 0.7 \\
\hline Time to surgery (y), median (IQR) & $2.8(1.3-4.4)$ & $4.1(1.8-5.8)$ & 0.1 \\
\hline \multicolumn{4}{|l|}{ Treatment history, n (\%) } \\
\hline Corticosteroids at enrollment & 9/38 (24\%) & $9 / 17(53 \%)$ & 0.033 \\
\hline Anti-TNF $\alpha$ at enrollment & $73 / 130(56 \%)$ & $28 / 37(76 \%)$ & 0.032 \\
\hline Immunomodulators & $115 / 135(85 \%)$ & $31 / 40(80 \%)$ & 0.39 \\
\hline Anti-TNF $\alpha$ exposure & $70 / 136(51 \%)$ & $27 / 40(69 \%)$ & 0.049 \\
\hline
\end{tabular}

$\mathrm{BMI}=$ body mass index $; \mathrm{CRP}=\mathrm{C}$-reactive protein $; \mathrm{ESR}=$ erythrocyte sedimentation rate; $\mathrm{GI}=$ gastrointestinal; $\mathrm{IQR}=$ interquartile range; $\mathrm{PA}=$ perianal; PGA $=$ Physician Global Assessment; SD = standard deviation; TNF $\alpha=$ tumor necrosis factor $\alpha$.

Italics represents $P<0.05$.

$(P=0.02)$. Overall there were 21 patients with fistulizing perianal disease confirmed by pMRI $(9 \%)$ and 36 patients $(15 \%)$ with skin tags/fissures (16 patients with skin tags only, 17 patients with skin tags and fissures, and 3 patients with fissures only) without an underlying fistula. Of patients with fistulizing perianal disease 10 patients had $>1$ fistula and 8 patients had perianal abscess during the study period.

\section{Cross-sectional Analysis}

In general, the presence of $\mathrm{pCD}$ was positively associated with most constructs of disease activity measured in the present study. Children with perianal findings had higher wPCDAI score, PGA of inflammatory activity, and global MRE inflammatory score (Fig. 1) compared with those without perianal involvement. Serum albumin and erythrocyte sedimentation rate similarly differed between the groups (Table 1). Children with pCD had reduced median weight $z$ scores $(-0.9$ [interquartile range $-1.9-0.2$ ] vs $-0.35[-1.3-0.5], P=0.03)$ and height $z$ scores $(-0.68[-1.4-0.2]$ vs $-0.23[-1.1-0.6], P=0.04]$ compared with non-pCD patients. Finally, pCD was associated with endoscopic disease severity of the UGI tract as measured by the UGI-simple endoscopic score for CD score, but not with ileocolonoscopy (Fig. 2).

Involvement of perianal disease was associated with the need for more aggressive treatment. More patients with perianal disease received corticosteroid treatment at enrollment (recorded in all patients regardless of disease duration at enrollment) and were exposed more often to anti-tumor necrosis factor $\alpha(\mathrm{TNF} \alpha)$ therapies (Table 1).

Those with $\mathrm{pCD}$ had different disease location with more jejunal (17/54 [31\%] vs 18/162 [11\%], $P=0.003)$ and rectal $(32 / 56$ [57\%] vs 67/176 [38\%], $P=0.04)$ involvement. Perianal disease was also associated with granulomatous disease (35/55 [64\%] vs 39/ $167[23 \%], P=0.001)$.

At enrollment, there was no difference between $\mathrm{pCD}$ and non-pCD patients in either the proportions of stricturing disease (15 [27\%] vs 50 [28\%], $P=0.9)$, or penetrating disease (5 [8\%] vs $9[5 \%], P=0.2)$ defined clinically or radiologically, respectively. Similarly, median PGA total tissue damage score $(19$ [5-32] vs 12 [4-25], $P=0.6)$ and MRE global damage scores (5 [1-20] vs 11 [2-31], $P=0.7$ ) did not differ between $\mathrm{pCD}$ and non-pCD patients, respectively. A total of $24(10 \%)$ patients underwent intestinal 


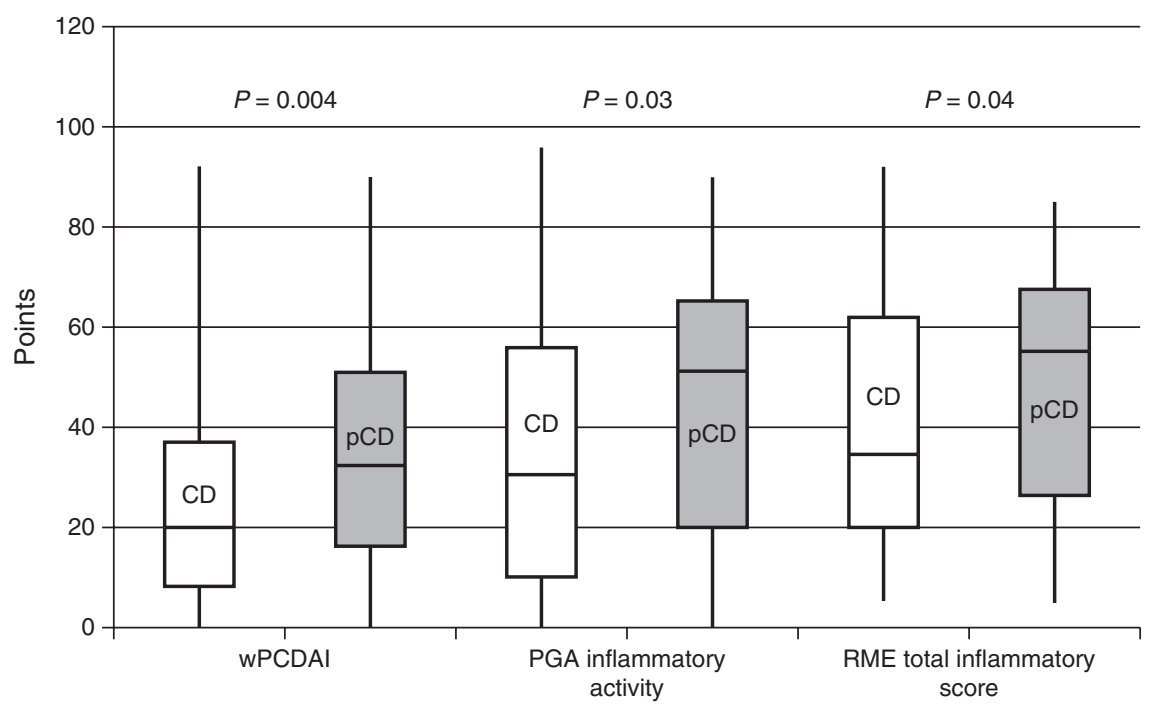

FIGURE 1. Clinical and radiological indices of patients with and without perianal disease. Analysis of 234 patients ( 177 with no perianal findings and 77 with perianal findings) shows that median WPCDAI, PGA inflammatory activity, and MRE total inflammatory scores significantly differ between groups (median: horizontal line, interquartile range [IQR]: box, range: vertical line, white boxes: non perianal, gray boxes: perianal). MRE = magnetic resonance enterography; $\mathrm{pCD}=$ perianal Crohn disease; $\mathrm{PGA}=$ Physician Global Assessment; $w P C D A I=$ weighted Pediatric CD Activity Index.

resection by the end of the follow-up period with no significant difference between the groups either in cumulative surgical rate (15 [8.5\%] vs 7, [12\%]; $P=0.5)$ or in time to resection.

A subgroup analysis of 21 children with confirmed perianal fistulae on pMRI showed similar significant differences most aforementioned outcomes (presence of granulomas, PGA of inflammatory activity, wPCDAI score, rectal and jejunal inflammation, global MRE inflammatory score, and need for anti-TNF $\alpha$ ) and lost significance regarding laboratory and anthropometric measures. In contrast, a subgroup analysis of 36 children who had skin tags or fissures without an underlying fistula compared with those without any perianal findings demonstrated no significant difference in any of the outcomes (Table 2), suggesting that fistulizing perianal disease contributed most to the differences in the outcomes.

\section{Longitudinal Analysis}

Ninety-eight children had their 18-month follow-up visit completed by the time of the data lock for the present study ( 56 with no perianal findings, 23 with skin tags/fissures only, and 19 with fistulizing perianal disease). Among those without perianal findings at enrollment, $3(5.3 \%)$ developed skin tags and $3(5.3 \%)$ developed

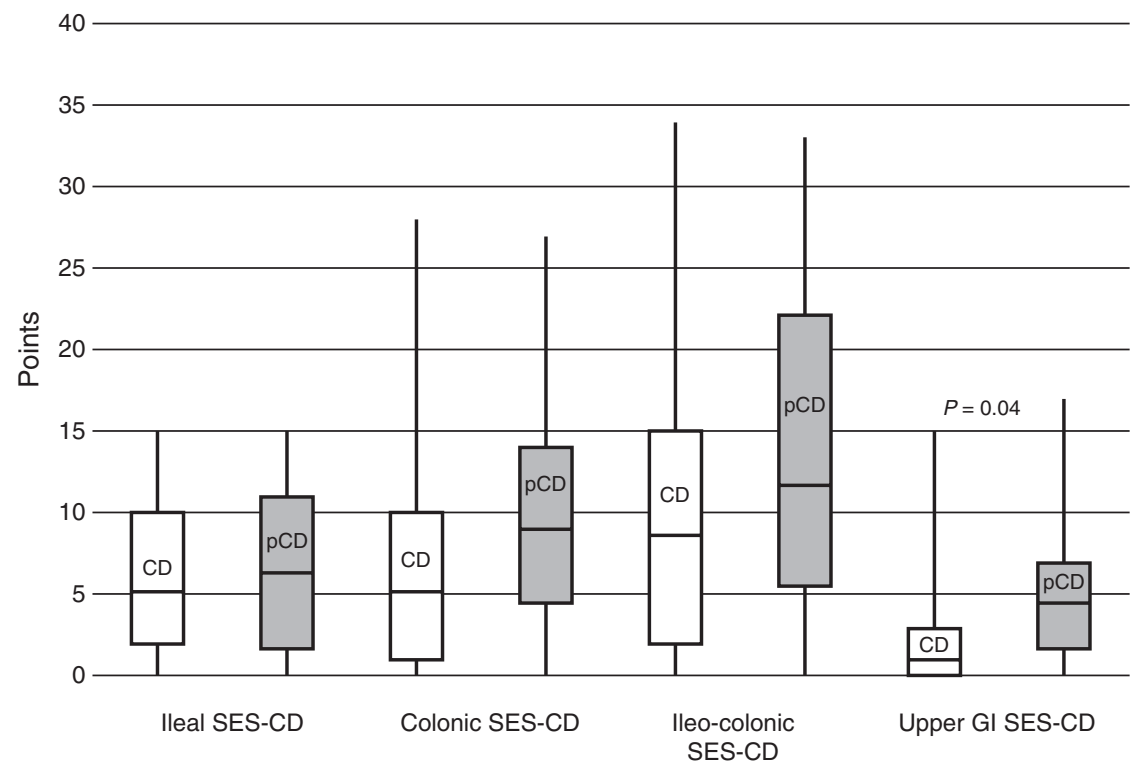

FIGURE 2. Endoscopic SES-CD of patients with and without perianal disease. Analysis of 234 patients ( 177 with no perianal findings and 77 with perianal findings) shows that only upper GI SES-CD differs between groups, whereas ileal, colonic, and ileocolonic do not. (median: horizontal line, interquartile range [IQR]: box, range: vertical line, white boxes: nonperianal, gray boxes: perianal). $\mathrm{Gl}=$ gastrointestinal; $\mathrm{pCD}=$ perianal $\mathrm{Crohn}$ disease; SES-CD = simple endoscopic score for Crohn disease. 
fistulizing disease. Only 1 patient with noninflamed skin tags at enrollment developed perianal fistula during follow-up. All patients with perianal fistulizing disease but 1 (18 patients, 95\%) were treated with anti-TNF $\alpha$. There was no difference in the proportion of patients treated with anti-TNF $\alpha$ between the 2 other groups (34 [61\%] patients with no findings compared with 12 [52\%] patients with skin tags, $P=0.6$ ). There was no difference in corticosteroids or immunomodulators' exposure between groups. Linear mixed models did not demonstrate significant differences between the 3 groups when analyzing the change in clinical variables, anthropometric measures, and radiologic scores (both inflammation and damage) between time 0 and 18 months.

Analysis of outcomes at 18 months demonstrated that both inflammatory PGA and global inflammatory MRE score improved significantly for the whole cohort $(40$ [18-63] to 10 [2-22], $P<0.001$ ]; 51 [20-66] to 28 [14-49], $P=0.002$ ) with no significant change in damage scores. The presence of perianal disease as classified in the aforementioned 3 groups did not alter the 18 months' measures including anthropometric measures, inflammatory global assessments, and damage scores at the end of follow-up.

\section{DISCUSSION}

In the present study, using the prospectively accrued data from the ImageKids cohort, we evaluated phenotypic and clinical features of pediatric perianal disease. As in adults, pediatric pCD was associated with worse clinical, laboratory, and anthropometric measures and with a higher burden of inflammatory activity. These patients had lower weight and height $z$ scores, lower serum albumin, higher erythrocyte sedimentation rate, and increased wPCDAI. The increased rate of active disease was also confirmed radiologically using MRE inflammatory scores. Furthermore, we observed a striking difference in jejunal involvement reflecting a higher inflammatory burden in pCD. Jejunal disease was previously demonstrated to be associated with poorer disease outcome in adult patients with CD (14). We also observed a higher rate of rectal involvement, consistent with findings previously reported in adult patients with $\mathrm{CD}(6,15)$ but contradicting others $(16)$. We found that inflamed skin tags may be associated with perianal fistulae detected by pMRI but not when tags are not inflamed. Finally, pCD was associated with higher prevalence of granulomas found in intestinal biopsy specimens as previously reported in adults (17). The presence of granulomas has also been associated with a more aggressive disease course (18).

Overall, only 4 pediatric studies compared pCD to non-pCD patients $(10,16,19,20)$. One study reported that pCD is associated with anorectal inflammation and tissue granulomata (19), and in the study by Keljo et al (16) it was associated with a greater use of immunomodulators and biologics. None of the studies reported an association with any other specific features suggesting a more severe phenotype (other than younger age at presentation and low body mass index (10)) or luminal complications.

Surprisingly, and in contrast to the increased inflammatory activity, perianal disease was not associated with intestinal damage as measured by radiologist global assessment of MRE, the presence of strictures and internal fistulae, and disease progression during 18 months. In contrast to our findings, previous studies performed in adults have shown that $\mathrm{pCD}$ predicted a more complicated disease course including internal fistulizing and stenotic disease $(6,7,15,21,22)$. In children, 2 studies reported a higher rate of intestinal resection in patients with pCD $(10,23)$, although the latter study was conducted before the introduction of biologics as an early treatment of pCD. It is plausible to assume that we did not observe such an association in our recent cohort because the presence of perianal disease prompted physicians to commence anti-TNF $\alpha$ agents as currently recommended (24), altering the natural history of the disease; $71 \%$ of those with pCD and $95 \%$ of those with fistulizing $\mathrm{pCD}$ were treated with anti-TNF $\alpha$. The increased usage of anti-TNF $\alpha$ thus likely merely reflects clinical practice rather than a reflection of a more severe disease. Similarly, the higher proportion of perianal patients treated with corticosteroids at enrollment may be attributed to the known diminished efficacy of exclusive enteral nutrition in perianal disease (24). Finally, intestinal resection rate was relatively low in our study, which may have hampered the power of prediction analyses.

Based on a comprehensive pMRI assessment we were able to stratify patients with perianal findings into those with skin tags and/ or fissures only compared to patients with confirmed fistulizing disease. The natural history of noninflamed skin tags is not well documented. In adults, a single study indicated that the natural history of skin tags or fissure is overall benign with most lesions being stable at 10 years of follow-up (25). Our study suggests that inflamed skin tags should be treated differently than noninflamed tags. Of the 32 patients with noninflamed skin tags in our study only 1 had an underlying fistula demonstrated radiologically in contrast to 3 of the 8 patients with inflamed skin tags. Even more reassuring is the fact that the risk for developing new fistulae during 18 months was similar between patients with noninflamed skin tags and with no perianal findings. Supporting this notion, in a subgroup analysis, only fistulizing perianal disease was associated with constructs of higher inflammatory activity, whereas skin tags and fissures were not.

The mechanism by which pCD confers greater risk for a more severe course is not well understood. The etiology of pCD seems to involve interaction between microbiological, immunological, and genetic factors (26). It is possible that the same genetic factors associated with severe or complicated disease such as Nucleotide-binding oligomerization domain-containing protein 2 (NOD2) also known as caspase recruitment domain-containing protein 15 (CARD15) and immunity-related GTPase family M

TABLE 2. Characteristics of Patients According to Specific Perianal Phenotype $(n=234)$

\begin{tabular}{|c|c|c|c|c|}
\hline & Non-PA $(\mathrm{n}=177)$ & Nonpenetrating PA $(\mathrm{n}=36)$ & Penetrating PA $(\mathrm{n}=21)$ & Significance $(P)$ \\
\hline Granuloma in histology, $\mathrm{n}(\%)$ & $39 / 167(23 \%)$ & $19 / 35(54 \%)$ & $16 / 20(75 \%)$ & 0.01 \\
\hline PGA disease activity (moderate-severe), n (\%) & $64(36 \%)$ & $17(47 \%)$ & $17(81 \%)$ & 0.01 \\
\hline MRE total inflammatory score, median (IQR) & $35(20-62)$ & $40(22-60)$ & $59(34-72)$ & 0.04 \\
\hline Rectal inflammation, n (\%) & $67 / 176(38 \%)$ & $15 / 35(43 \%)$ & $17 / 21(81 \%)$ & 0.02 \\
\hline Jejunal inflammation, n (\%) & $18 / 162(11 \%)$ & $8 / 34(24 \%)$ & $9 / 20(45 \%)$ & 0.01 \\
\hline
\end{tabular}

$\mathrm{IQR}=$ interquartile range; $\mathrm{MRE}=$ magnetic resonance enterography; $\mathrm{PA}=$ perianal; $\mathrm{PGA}=$ Physician Global Assessment; $\mathrm{wPCDAI}=$ weighted pediatric Crohn disease activity index.

Italics represents $P<0.05$. 
protein (IRGM) $(27,28)$ also predispose to perianal disease. Only few studies aimed at identifying genetic factors associated with pCD, but most were limited by small sample size. An association of pCD with a susceptibility locus on chromosome 5 has been described (5q31) (29,30); however, other genetic association studies have confirmed neither these findings $(31,32)$.

This is one of only a few studies assessing phenotypic and clinic characteristics of pediatric pCD. Our results are strengthened by the prospective nature of the study and by the comprehensive analyses of clinical, laboratory, anthropometric, endoscopic, and radiologic measures. Although prospective in design, the present study has several limitations. First, despite being one of the largest studies evaluating perianal disease in children, the number of children with perianal involvement within the cohort is relatively small and therefore findings may be at risk of type II error. Moreover, patients were enrolled at different time points during disease course, confounding the longitudinal assessment. On the contrary, the differing disease duration allowed us to explore the phenotype of pediatric pCD during the course of disease.

In conclusion, pediatric $\mathrm{pCD}$ has distinct phenotypic features and a greater inflammatory burden during the course of disease, which is mainly driven by fistulizing disease rather than noninflamed skin tags or fissures. In the era of standard early anti-TNF $\alpha$ treatment for perianal disease, we did not observe higher rates of intestinal damage associated with this phenotype. Therefore, our findings support the notion that the presence of fistulizing perianal disease reflects a more severe disease and thus warrants considering more aggressive management approach. Isolated noninflamed skin tags and/or fissures may not act similarly as a predictive variable and thus may not require a radiologic evaluation or treatment escalation.

Acknowledgments: The authors thank Dr Tomer Ziv (PhD), statistician, Tel-Aviv University, Tel-Aviv, Israel for performing statistical analysis.

For the ImageKids collaborators, please see Supplemental Digital Content, Appendix, http://links.lww.com/MPG/A863.

\section{REFERENCES}

1. Lehtinen $\mathrm{P}$, Ashorn $\mathrm{M}$, Iltanen $\mathrm{S}$, et al. Incidence trends of pediatric inflammatory bowel disease in Finland, 1987-2003, a nationwide study. Inflamm Bowel Dis 2011;17:1778-83.

2. Abraham BP, Mehta S, El-Serag HB. Natural history of pediatric-onset inflammatory bowel disease: a systematic review. J Clin Gastroenterol 2012;46:581-9.

3. De Bie CI, Paerregaard A, Kolacek S, et al. Disease phenotype at diagnosis in pediatric Crohn's disease: 5-year analyses of the EUROKIDS Registry. Inflamm Bowel Dis 2013;19:378-85.

4. Hellers G, Bergstrand O, Ewerth S, et al. Occurrence and outcome after primary treatment of anal fistulae in Crohn's disease. Gut 1980;21:525-7.

5. Schwartz DA, Loftus EV, Tremaine WJ, et al. The natural history of fistulizing Crohn's disease in Olmsted County, Minnesota. Gastroenterology 2002;122:875-80.

6. Tang LY, Rawsthorne P, Bernstein CN. Are perineal and luminal fistulas associated in Crohn's disease? A population-based study. Clin Gastroenterol Hepatol 2006;4:1130-4.

7. Tarrant KM, Barclay ML, Frampton CMA, et al. Perianal disease predicts changes in Crohn's disease phenotype: results of a population-based study of inflammatory bowel disease phenotype. Am $J$ Gastroenterol 2008;103:3082-93.

8. Beaugerie L, Seksik P, Nion-Larmurier I, et al. Predictors of Crohn's disease. Gastroenterology 2006;130:650-6.

9. Lapidus A, Bernell O, Hellers G, et al. Clinical course of colorectal Crohn's disease: a 35-year follow-up study of 507 patients. Gastroenterology 1998;114:1151-60.

10. Short SS, Dubinsky MC, Rabizadeh S, et al. Distinct phenotypes of children with perianal perforating Crohn's disease. J Pediatr Surg 2013;48:1301-5.
11. Levine A, Koletzko S, Turner D, et al., European Society of Pediatric Gastroenterology, Hepatology, and Nutrition. ESPGHAN revised porto criteria for the diagnosis of inflammatory bowel disease in children and adolescents. J Pediatr Gastroenterol Nutr 2014;58:795-806.

12. Levine A, Griffiths A, Markowitz J, et al. Pediatric modification of the Montreal classification for inflammatory bowel disease: The Paris classification. Inflamm Bowel Dis 2011;17:1314-21.

13. Ledder O, Church P, Griffiths AM, et al. Utility of proposed modified simple endoscopic score in upper gastrointestinal Crohn's disease (abstract). SZMC Research Conference, 2016.

14. Lazarev M, Huang C, Bitton A, et al. Relationship between proximal Crohn's disease location and disease behavior and surgery: a crosssectional study of the IBD Genetics Consortium. Am J Gastroenterol 2013;108:106-12.

15. Kaur M, Panikkath D, Yan X, et al. Perianal Crohn's disease is associated with distal colonic disease, stricturing disease behavior, IBD-associated serologies and genetic variation in the JAK-STAT pathway. Inflamm Bowel Dis 2016;22:862-9.

16. Keljo DJ, Markowitz J, Langton C, et al. Course and treatment of perianal disease in children newly diagnosed with Crohn's disease. Inflamm Bowel Dis 2009;15:383-7.

17. Denoya P, Canedo J, Berho M, et al. Granulomas in Crohn's disease: does progression through the bowel layers affect presentation or predict recurrence? Colorectal Dis 2011;13:1142-7.

18. Heresbach D, Alexandre JL, Branger B, et al., ABERMAD (Association Bretonne d'Etude et de Recherche sur les Maladies de l'Appareil Digestif). Frequency and significance of granulomas in a cohort of incident cases of Crohn's disease. Gut 2005;54:215-22.

19. Markowitz J, Daum F, Aiges H, et al. Perianal disease in children and adolescents with Crohn's disease. Gastroenterology 1984;86:829-33.

20. Zwintscher NP, Shah PM, Argawal A, et al. The impact of perianal disease in young patients with inflammatory bowel disease. Int $J$ Colorectal Dis 2015;30:1275-9.

21. Sachar DB, Bodian CA, Goldstein ES, et al. Is perianal Crohn's disease associated with intestinal fistulization? Am J Gastroenterol 2005; 100:1547-9.

22. Thia KT, Sandborn WJ, Harmsen WS, et al. Risk factors associated with progression to intestinal complications of Crohn's disease in a population based cohort. Gastroenterology 2010;139:1147-55.

23. Gupta N, Cohen SA, Bostrom AG, et al. Risk factors for initial surgery in pediatric patients with Crohn's disease. Gastroenterology 2006;130:1069-77.

24. Ruemmele FM, Veres G, Kolho KL, et al., European Crohn's, Colitis Organisation, European Society of Pediatric Gastroenterology, Hepatology, Nutrition. Consensus guidelines of ECCO/ESPGHAN on the medical management of pediatric Crohn's disease. J Crohns Colitis 2014;8:1179-207.

25. Buchmann P, Keighley MR, Allan RN, et al. Natural history of perianal CD. Ten year follow-up: a plea for conservatism. Am J Surg 1980;140:642-4.

26. Tozer PJ, Whelan K, Phillips RK, et al. Etiology of perianal Crohn's disease: role of genetic, microbiological, and immunological factors. Inflamm Bowel Dis 2009;15:1591-8.

27. Schnitzler F, Friedrich M, Wolf C, et al. The NOD2 p.Leu1007fsX1008 mutation (rs2066847) is a stronger predictor of the clinical course of Crohn's disease than the FOXO3A intron variant rs 12212067. PLoS One 2014;9:e108503.

28. Klausz G, Molnár T, Nagy F, et al. Polymorphism of the heat-shock protein gene Hsp70-2, but not polymorphisms of the IL-10 and CD14 genes, is associated with the outcome of Crohn's disease. Scand J Gastroenterol 2005;40:1197-204.

29. Armuzzi A, Ahmad T, Ling KL, et al. Genotype-phenotype analysis of the Crohn's disease susceptibility haplotype on chromosome 5q31. Gut 2003;52:1133-9.

30. Onnie CM, Fisher SA, Prescott NJ, et al. Diverse effects of the CARD15 and IBD5 loci on clinical phenotype in 630 patients with Crohn's disease. Eur J Gastroenterol Hepatol 2008;20:37-45.

31. Weersma RK, Stokkers PC, Van Bodegraven AA, et al. Molecular prediction of disease risk and severity in a large Dutch Crohn's disease cohort. Gut 2009;58:388-95.

32. Brant SR, Picco MF, Achkar JP, et al. Defining complex contributions of NOD2/CARD15 gene mutations, age at onset, and tobacco use on Crohn's disease phenotypes. Inflamm Bowel Dis 2003;9:281-9. 